\title{
Manufacturing of Alumina-Zirconia-CNT Ceramic Composites: A comparison of Conventional and Alumina-Graphite Powder Bath Sintering methods
}

Diwakar Makireddi ( $\sim$ diwakar.makireddi@gmail.com )

VNIT: Visvesvaraya National Institute of Technology

\section{D Ghuge}

Visvesvaraya National Institute of Technology

\section{ALOK BEHERA}

Indian Institute of Science https://orcid.org/0000-0001-5485-2303

Y M Puri

Visvesvaraya National Institute of Technology

\section{Gajanan Thakare}

Castwel Industries

\section{M Thawre}

Visvesvaraya National Institute of Technology

\section{Research Article}

Keywords: Ceramic-matrix composites (CMCs), Hardness, Microstructural analysis, Sintering

Posted Date: October 25th, 2021

DOl: https://doi.org/10.21203/rs.3.rs-968566/v1

License: (1) This work is licensed under a Creative Commons Attribution 4.0 International License.

Read Full License 


\title{
Manufacturing of Alumina-Zirconia-CNT Ceramic Composites: A comparison of Conventional and Alumina-Graphite Powder Bath Sintering methods
}

\author{
Diwakar Makireddi $^{1 *}$, V D Ghuge ${ }^{1}$, Alok Behera $^{2}$, Y M Puri ${ }^{1}$, Gajanan $_{\text {Thakare }}^{3}$, M M \\ Thawre $^{4}$ \\ ${ }^{1}$ Department of Mechanical Engineering, Visvesvaraya National Institute of Technology, \\ Nagpur, Maharastra-440010, India \\ ${ }^{2}$ Department of Mechanical Engineering, Indian Institute of Science, Bangalore, Karnataka- \\ 560012 , India \\ ${ }^{3}$ Castwel Industries, MIDC, Nagpur, Maharastra-440010, India \\ ${ }^{4}$ Department of Metallurgical and Materials Engineering, Visvesvaraya National Institute of \\ Technology, Nagpur, Maharastra-440010, India \\ *Corresponding author E-mail: diwakar.makireddi@gmail.com
}

\begin{abstract}
The Zirconia $\left(\mathrm{ZrO}_{2}\right)$ and Carbon Nano Tubes (CNTs) embedded Alumina $\left(\mathrm{Al}_{2} \mathrm{O}_{3}\right)$ composite fabricated using conventional sintering (CS) possess higher strength, better micro-hardness and more toughness as compared to pure alumina. However, several researchers have reported nonuniform mechanical properties in CS. This non-uniformity can be eliminated using the novel Alumina-Graphite Powder Bath (AGPB) sintering technique. In AGPB, the alumina composites are surrounded by an equal proportion mixture of alumina-graphite powders, providing indirect uniform heating. This converts the heat transfer mode from convectionradiation to conduction and produces uniform mechanical properties across the sintered surface. In this paper, both AGPB and CS techniques were used to fabricate Alumina-ZirconiaCNT (AZC) composites. The uniformity of Physico-mechanical properties was compared for AGPB-AZC and CS-AZC composites. The AGPB-AZC composites exhibited better hardness and minimum pore dispersion as compared to CS-AZC composites. The AGPB-AZC FESEM analysis revealed the formation of single-crystal structures across the sintered surface, which eventually improved the hardness. The mean hardness of the AGPB-AZC (1829.95 HV) composite was enhanced by $43 \%$ compared to CS-AZC (1273.5 HV).
\end{abstract}

Keywords: Ceramic-matrix composites (CMCs), Hardness, Microstructural analysis, Sintering 


\subsection{Introduction}

During the sintering process, the ceramic particles are bonded by mass transport mechanisms at working temperatures to reduce surface energies, which further affects significant grain growth, densification, and pore reduction. Conventional Sintering (CS) is a preferred technique for fabricating industrial ceramics like alumina composites, silicon carbide, zirconium dioxide, cordierite tungsten carbide, and mullite. Manufacturing these is challenging due to non-uniform heating and massive heat loss in the furnace [1]. Researchers have tried many modified CS techniques to reduce the associated heat loss and improve the mechanical properties [2]. The improvised CS techniques like two-step sintering (TSS) employ heating at two temperatures (High and Low temperatures), thus providing better control over the heating process. The twostage heating controls grain boundary migration and grain boundary diffusion.[2][3]. Even though this technique regulates abnormal grain growth and produces dense structures, researchers have still reported non-uniform mechanical properties across the sintered surface. The green ceramics in CS are heated by hot gases (atmospheric air/inert gas) through radiation and convection, preventing even heating across the surface due to the turbulent hot gases. As a result, researchers have reported density and mechanical property gradients in sintered ceramics. Gremillard et al.[4] fabricated zirconia-toughened alumina composites and studied the temperature gradients among the surface and center of a sphere specimen. They found significant temperature gradients that lead to density gradients, which further causes strain gradients. The strain gradient acts as a defect and can lead to premature fracture. Green et al. [5] found density gradients that occurred due to inhomogeneous powder packing and sintering rate mismatch, which further developed internal stresses.

Apart from the green body defects in CS, the convection-radiation mode also causes uneven heat supply, resulting in differential sintering, as shown in Fig.1 (a). Furthermore, the low thermal conductivity of ceramics creates thermal gradients across the sinter. Hence, uniform input heat energy is essential to maintain 'uniform diffusional sintering mechanisms' across the specimen volume. This constant heating can be attained using the novel AluminaGraphite Powder Bath (AGPB) sintering technique which converts convection-radiation mode into conduction mode. A schematic of AGPB sintering showing conduction mode heat transfer is shown in Fig. 1 (b). Fellah et. al.[6] fabricated $\alpha$-alumina ceramics using 'pure alumina powder bath' sintering technique and reported improved densification. Even though this technique improves densification, issues like the formation of amorphous structure, high porosity, and uneven hardness still exist. This even hampers the mechanical properties and 
usability during the working environment. Makireddi et. al.[7] compared the pure alumina powder bath and AGPB sintering techniques through hardness mapping and microstructural characterization. They reported significant improvements in grain boundary diffusion and uniform hardness across the sintered surface.
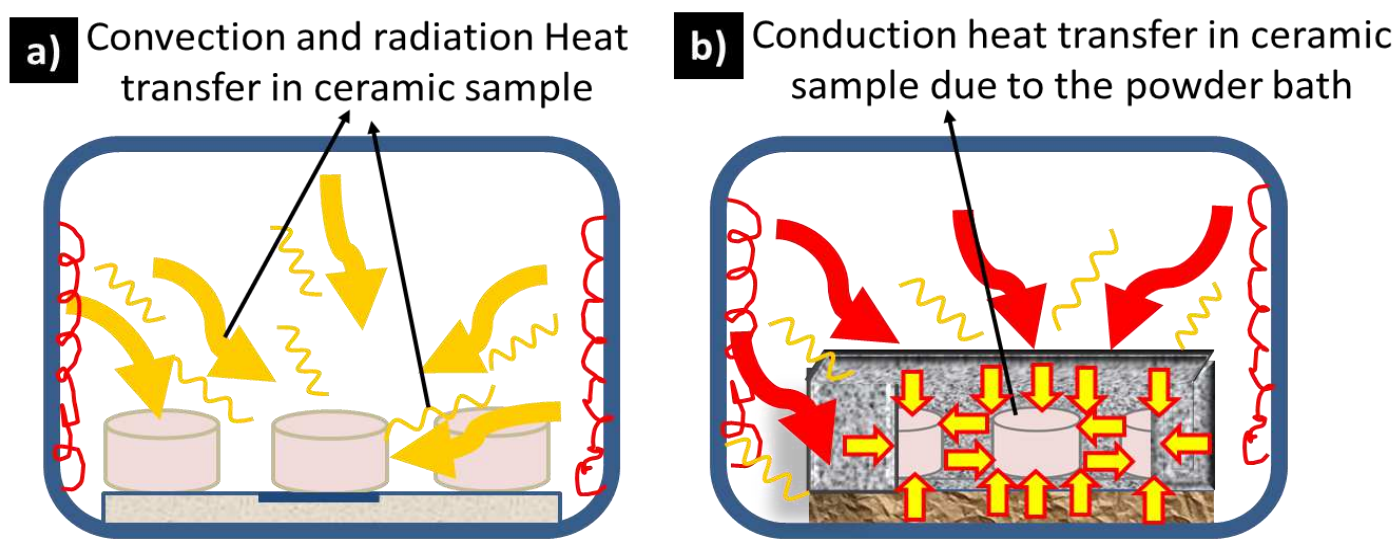

Fig. 1(a) Classical sintering process, (b) Powder bath sintering process of alumina-based ceramics

Zirconia has various applications such as electrolytes in manufacturing solid oxide fuel cells, biomedical applications, orthopaedic implants, dental, cutting tools, and thermal barrier coating, etc. [8]. The addition of Zirconia in alumina Composites increases the corrosion and wear resistance and its biocompatibility. Benzaid et al. [9] fabricated a Zirconia-alumina nanocomposite for medical applications and examined the fracture toughness, strength, and crack growth phenomenon. The fabricated alumina toughened Zirconia exhibited better aging resistance in comparison to the tetragonal metastable phase. Similarly, adding Zirconia $\left(\mathrm{ZrO}_{2}\right)$ in alumina increases the fracture toughness due to the tetragonal to monoclinic phase transformation. This causes volumetric expansion resulting in increased internal stresses, which further minimizes the possibilities of crack propagation in the ceramic matrix.

Like Zirconia, carbon nanotubes (CNTs) are a preferred additive among researchers for several ceramic composites. The CNT offers excellent chemical and mechanical properties for several applications. Ma et al. fabricated CNT (10 wt.\%)-silicon carbide (SiC) composites using the hot-pressing method. A $10 \%$ increase in fracture toughness and mechanical strength was observed compared to monolithic ceramics [10]. Even though ceramics possess high 
stiffness and better thermal stability, the associated brittleness restricts its application as a structural material. This can be avoided by using special resilient CNT additives in the ceramic composite. The CNT and alumina ceramic blend can potentially produce an exceptional composite with high-temperature stability, toughness, and creep resistance. The combined addition of Zirconia and CNT as additives in alumina ceramic has not been explored much. Hence, the sinterability of the Zirconia-CNT Toughened Alumina Composite using the novel AGPB sintering technique is studied here. The effects of the excellent uniform heating mechanism associated with AGPB techniques on the sintered composite are evaluated using certain mechanical and microstructural characterization.

\subsection{Experimental Procedure}

\subsection{Starting Materials}

Commercially available alumina (APS: $2-4 \mu \mathrm{m}$, Purity>96.8\%) and graphite (APS:100-325 mesh, Purity $>99.9 \%$ ) powders supplied by Loba Chemie, India, were used as starting materials for powder bath preparation. For green compacts preparation, commercially available $\alpha-\mathrm{Al}_{2} \mathrm{O}_{3}$ (APS:100-325 Mesh, Calcinated, Purity> 99.9\%, Loba Chemie, India), $\mathrm{ZrO}_{2}$ (APS:11-39 $\mu \mathrm{m}$, Purity > 99.9\%, Loba Chemie, India), and Carbon Nanotubes (CNTs, AdNano Technologies Pvt. Ltd) were used. The mean outer diameter, inner diameter, and length of CNTs are 20-30 $\mathrm{nm}, 10-20 \mathrm{~nm}$, and 20-30 $\mu \mathrm{m}$, respectively.

\subsection{Mixture and Processing}

The green compact was prepared using eight weight $\%$ of $\mathrm{ZrO}_{2}$, two weight $\%$ CNTs, and the rest with Alumina matrix. Poly Vinyl Alcohol (PVA) which generally gets evaporated approximately at $600{ }^{\circ} \mathrm{C}$ (well below working temperature), was used as a temporary binder to strengthen the green compacts [11]. The ceramic powders were homogeneously grinded using a mortar-pestle set. Then, the cylindrical pellets were compacted using a 6-ton load in a laboratory hydraulic press (Make: KIMAYA Engineers, Capacity: 15 tons). The cylindrical compacts were of $12 \mathrm{~mm}$ diameter and $7 \mathrm{~mm}$ height.

For preparing the AGPB sintering medium, an equal volume percentage of alumina and graphite powders were stirred homogeneously for an hour. The green compacts were entirely covered with this powder bath such that the pellets should not be visible outside, as shown in Fig. 1 (b). These arrangements were kept on a refractory brick, as shown in Fig. 2. The green pellets were sintered in a high-temperature muffle furnace (Make: Dass \& Co,1650 ${ }^{\circ} \mathrm{C}$ ) using a well-designed sintering cycle. 


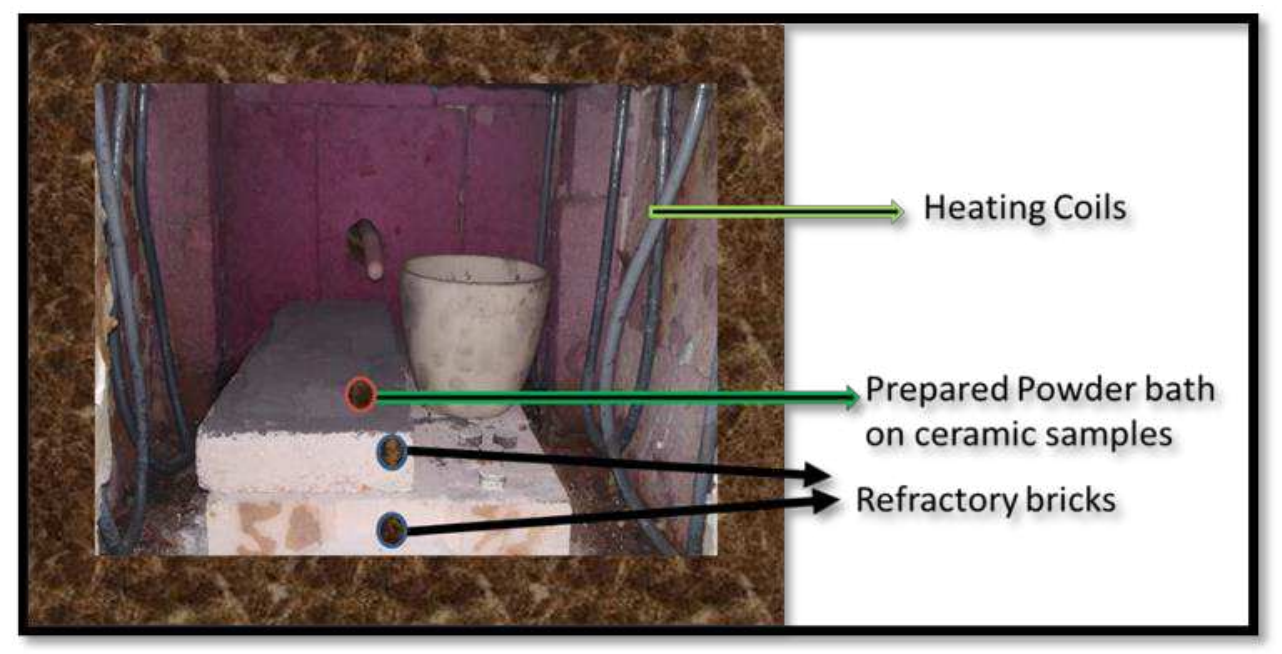

Fig. 2 Powder bath sintering arrangements in furnace

\section{3. sintering process}

The placement or positioning of green compacts in powder bath sintering is vital to achieving uniform heating across all directions. The sintered ceramics placed at the edges did not exhibit rigid structures during the trial-and-error process, as shown in Fig. 3 (a). The uneven distribution of powder baths around the edge samples leads to an inconsistent heat supply. Hence, the specimens should be precisely positioned at the center of the powder bath, as shown in Fig. 3 (b). It ensures that the green compacts observe the heat uniformly from all directions throughout the sintering process.
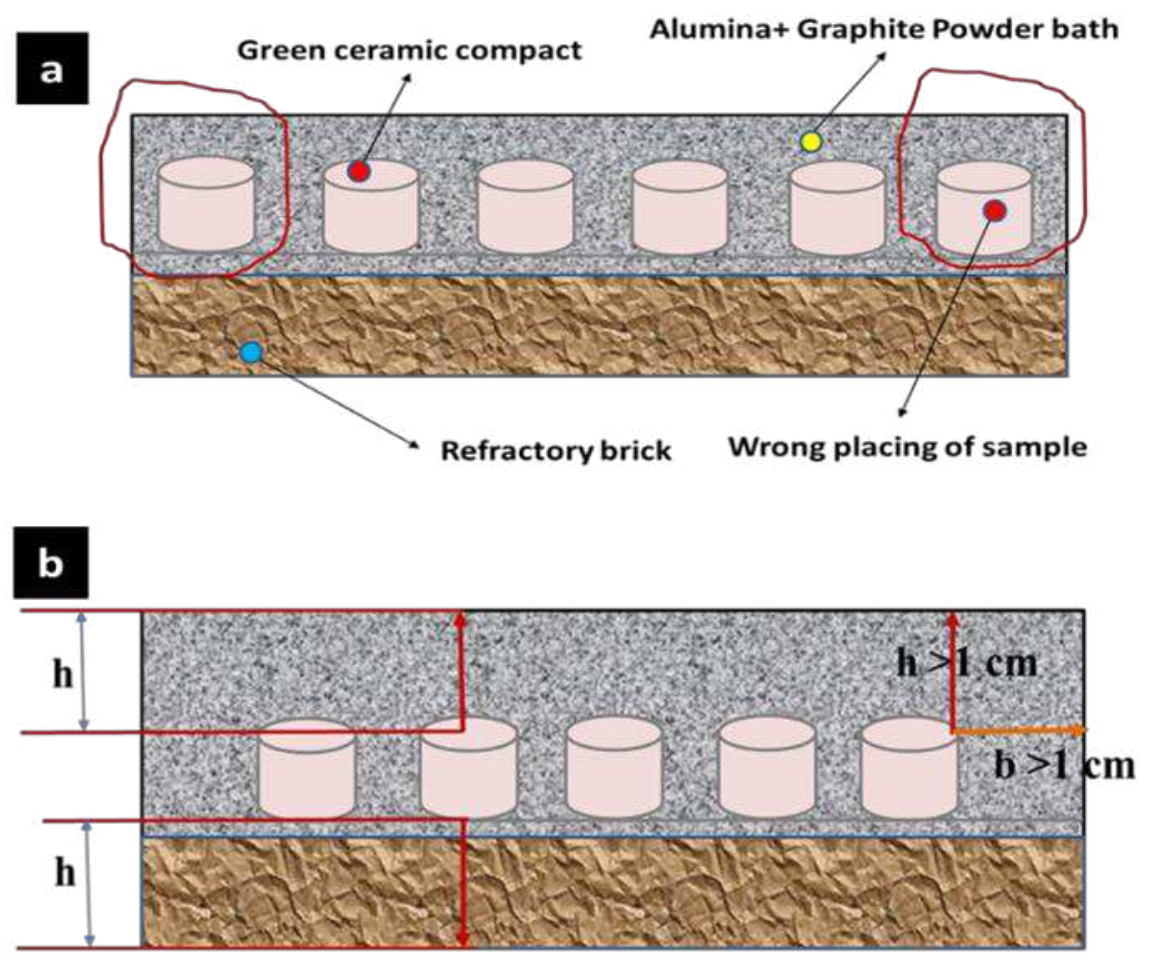
Fig. 3 (a) wrong positioning (b) accurate positioning of green compacts inside the powder bath

The AGPB sintering process has three primary steps, as shown in Fig. 4. In step one, a thin layer ( 2 to $4 \mathrm{~mm}$ ) of alumina-graphite powder bath was spread over the refractory brick, and the green compact was gently placed over it. In step two, the powder bath is distributed uniformly around the green compact. In step three, this setup was kept in a furnace for the sintering process. The CS-AZC and AGPB-AZC cylindrical specimens had a diameter of 12 $\mathrm{mm}$ and a height of $7 \mathrm{~mm}$, as shown in Fig. 5.
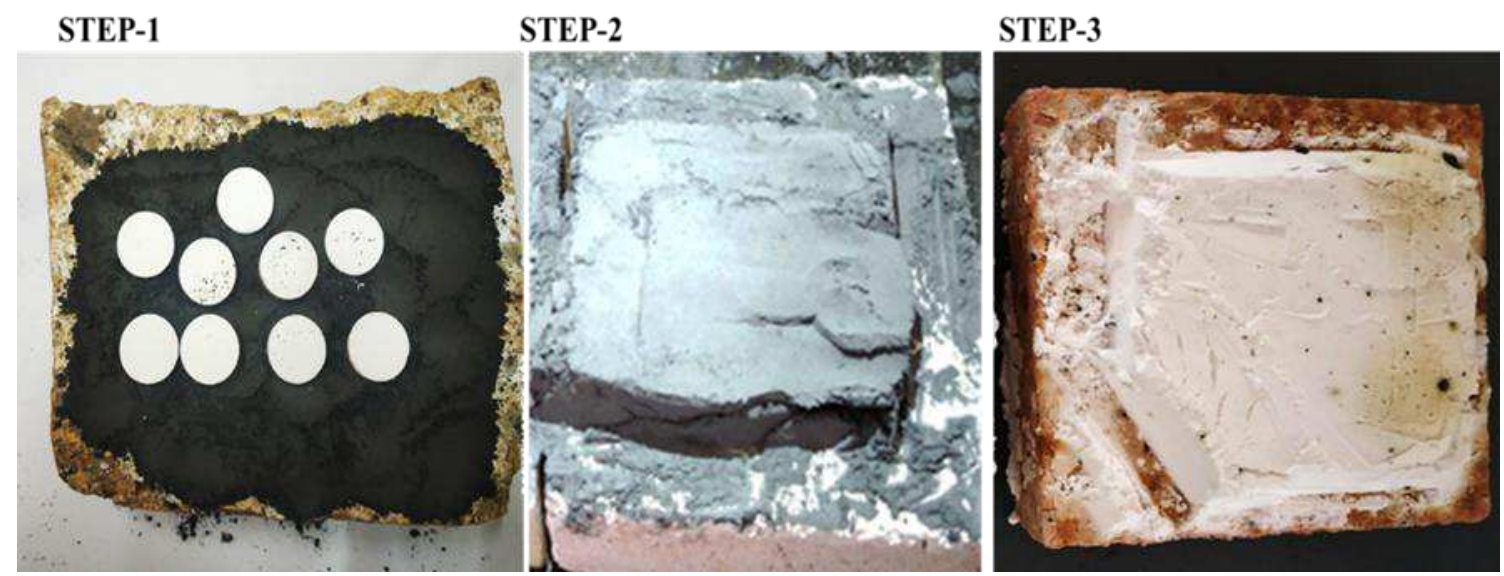

Fig. 4 Workflow in AGPB sintering process

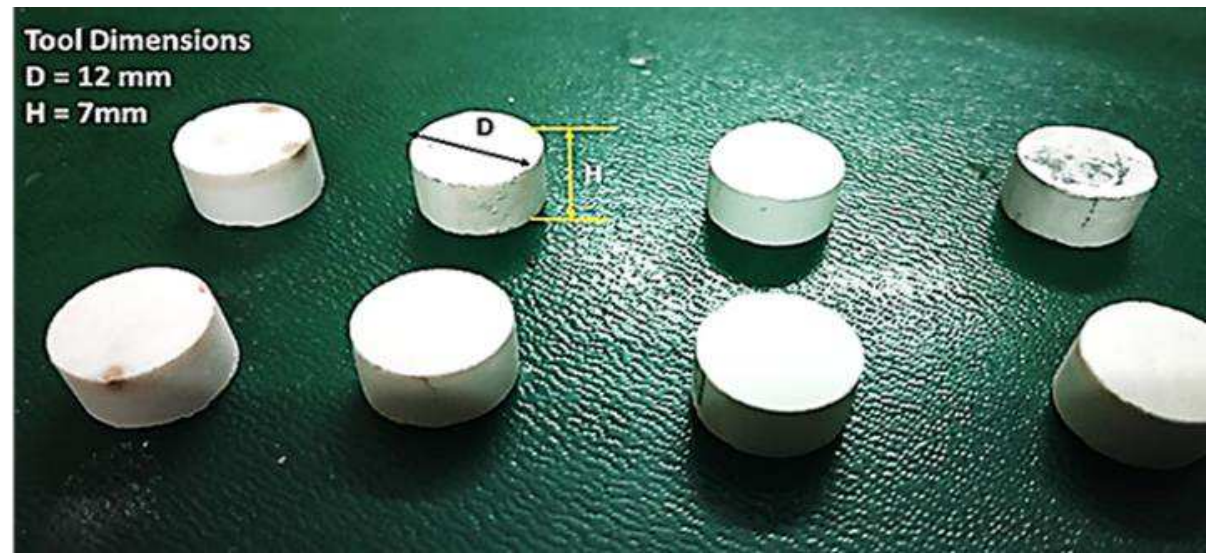

Fig. 5 AZC composite specimens

\subsection{Sintering Cycle}

The sintering parameters for CS-AZC and AGPB-AZC are schematically shown in Fig. 6. Initially, the high step temperature $\left(\mathrm{T}_{1}=1650^{\circ} \mathrm{C}\right)$ was achieved by a heating rate of $5{ }^{\circ} \mathrm{C} / \mathrm{min}$ from atmospheric temperature. A high step holding time of $t_{1}=2 \mathrm{~h}$ was maintained before moving down to a low step temperature of $\mathrm{T}_{2}=1500{ }^{\circ} \mathrm{C}$. The low step temperature holding time was $t_{2}=4 \mathrm{~h}$, as shown in Fig. 6. Generally, ceramics are poor in thermal conductivity, and a 
higher heating rate can cause cracked surfaces. Hence, a comparatively lower heating rate of 5 ${ }^{\circ} \mathrm{C} / \mathrm{min}$ was used to avoid thermal expansion mismatch between the surface and centre of the specimen. AGPB sintering typically consists of two heating curves: convection-radiation heating of powder bath followed by conduction mode uniform heating of green compacts as illustrated in Fig. 6. The green compacts receive heat from the high-temperature powders, thus converting the heating mode from convection-radiation to conduction.

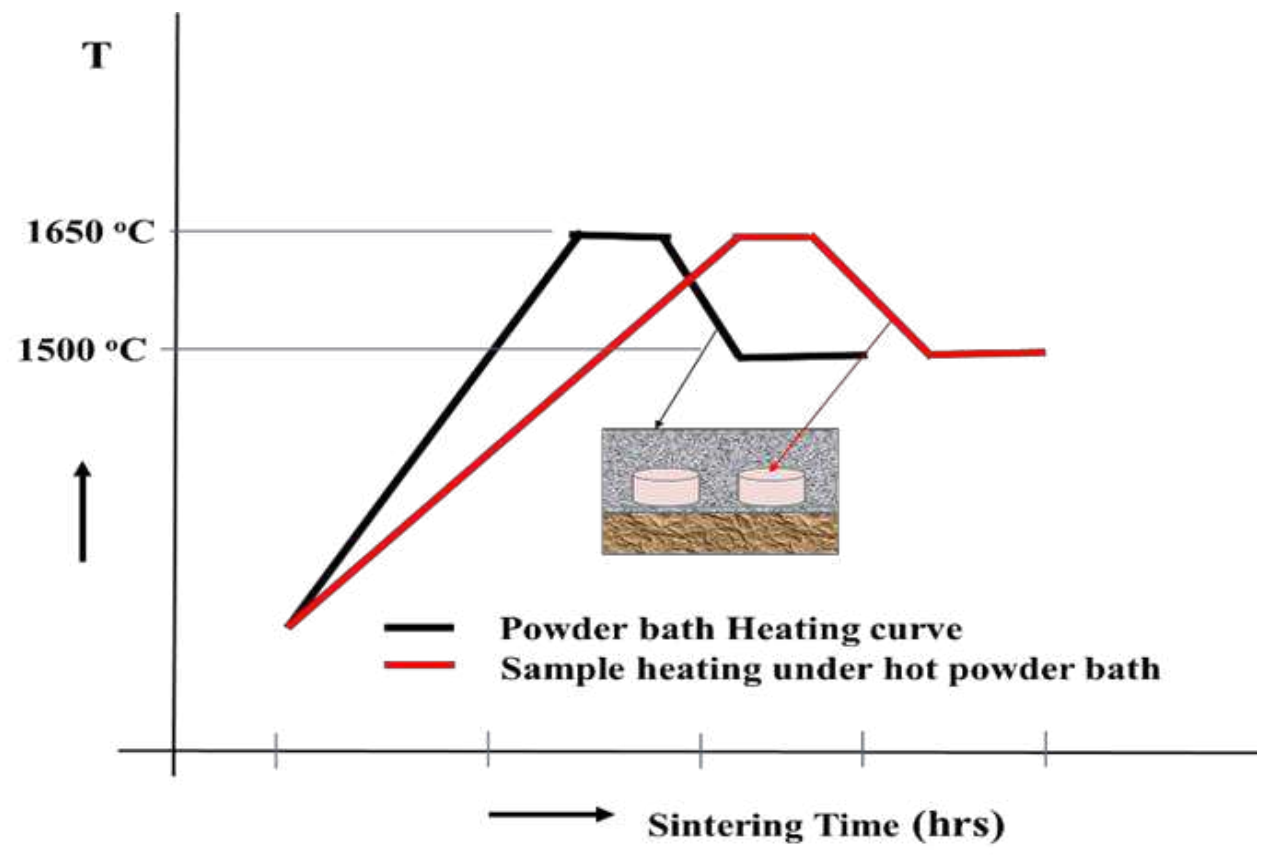

Fig. 6 Heating cycle for AGPB-AZC sintering

\subsection{Mechanical and Structural Characterization}

Micro Vickers Hardness (Make: Mitutoyo, Model: HM 200) was used to measure the surface hardness of AZC composites using a $10 \mathrm{~N}$ load with a dwell time of $16 \mathrm{~s}$ [12]. The average of a minimum of three hardness readings per location is reported further. Theoretical density was calculated using the rule of the mixtures, and the experimental density was measured using Archimedes' principle. The microstructure characterization of polished specimens was carried out using a Field Emission Scanning Electron Microscope (Make: FESEM- JEOL JSM 7610 F). Energy Dispersive Spectroscopy (EDS) was used to analyze the elemental distributions.

\subsection{Results and Discussion}

\subsection{Microstructure of as-sintered AZC composites}


The as-sintered CS and AGPB specimens were characterized to understand the effect of the heating on the evolved microstructure. The specimens were polished and platinum-coated before FESEM characterization. The FESEM micrographs and EDS analysis confirmed the proper distribution of constituent elements like $\mathrm{ZrO}_{2}$ and CNTs across the matrix for CS and AGPB techniques. A CS-AZC micrograph with EDS analysis is presented in Fig. 7.

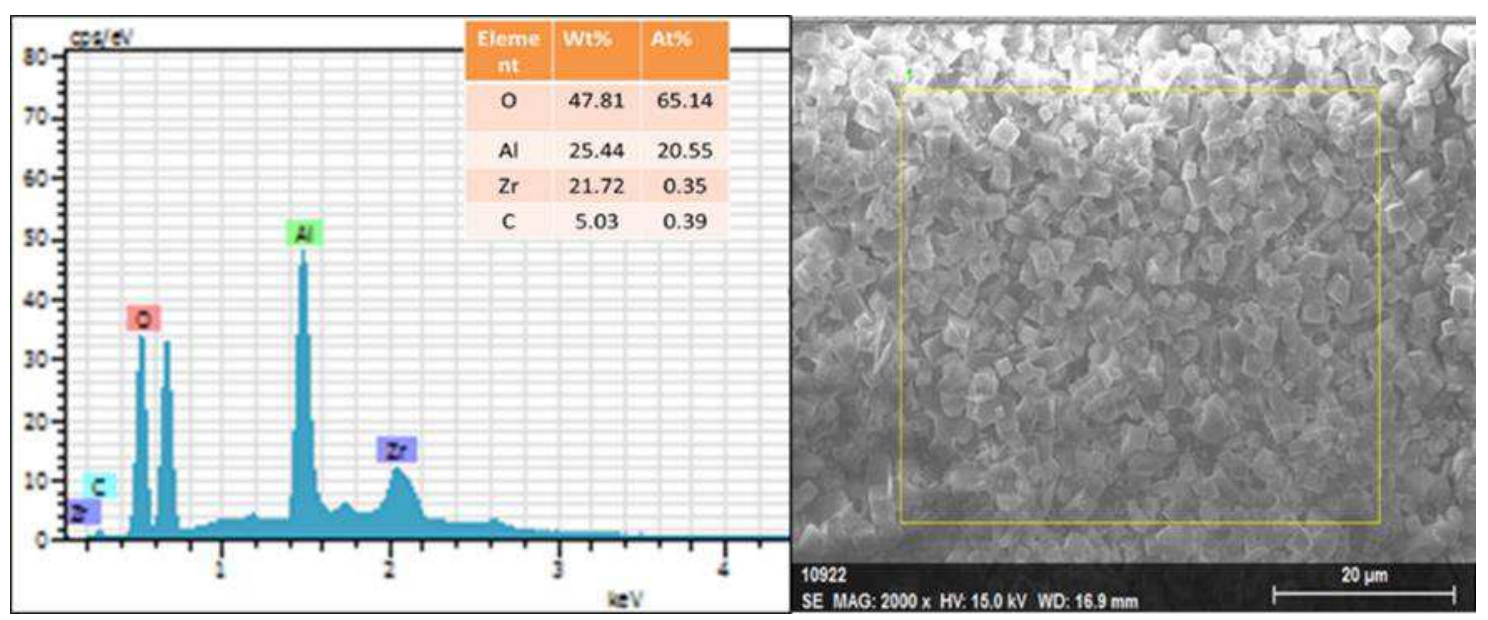

Fig. 7 Elemental analysis of AZC composites using EDS

The embedded CNT, diffused grains, few densified matrix regions along with porosity were observed, as shown in Fig. 8 (a-f). The noticeable individual particles of alumina, Zirconia, and CNTs reflect insignificant and improper bonding with the matrix.
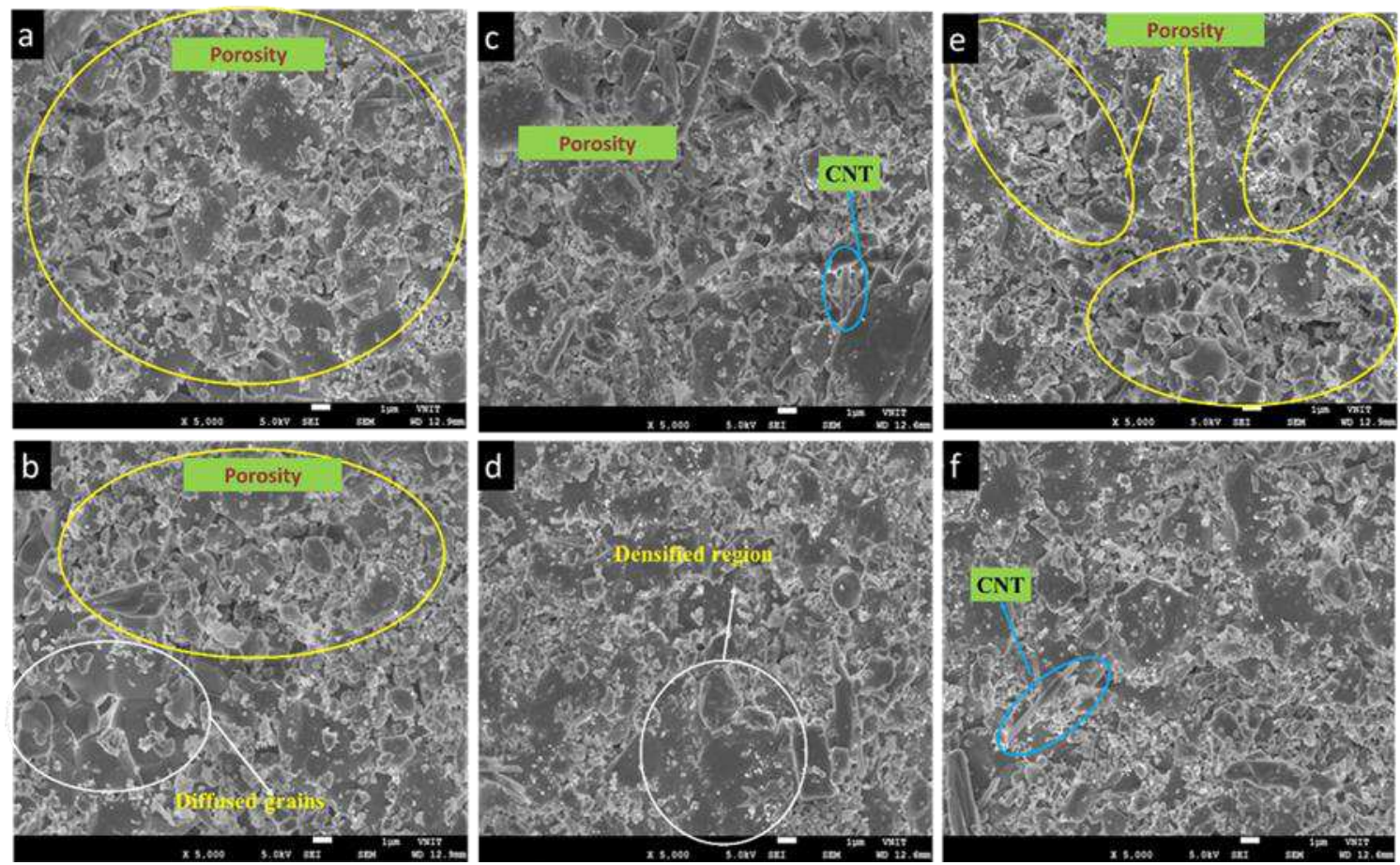

Fig. 8 FESEM micrographs of as-sintered CS-AZC specimen 
The CS-AZC microstructures showed significant porosity scattered throughout the sintered surface. This lack of fibre/matrix bonding and scattered porosity ultimately results in nonuniform mechanical properties across the surface. However, adequately diffused grains and densified regions with nominal porosity were observed in few regions, which is again a consequence of non-uniform heating.

The AGPB technique with uniform heating resulted in single-crystal structures, as shown in Fig. 9 (a-f). The AGPB-AZC revealed fully densified grain structures with negligible dispersed porosity as compared to CS-AZC. Efficient heat input in AGPB-AZC leads to an effective diffusion process across the surface during working temperatures. The sintering mechanism used in AGPB-AZC and CS-AZC specimens was remarkably different. In AGPB sintering, the specimens were heated through the surrounding alumina-graphite powder bath, where the convection-radiation heat transfer mode was converted to conduction mode inside the furnace. Traditionally, conduction mode heat transfer is more efficient, uniform, and consistent over time than convection/radiation. This demonstrates the conversion of nonuniform to uniform heating of specimen from all the direction through AGPB technique.
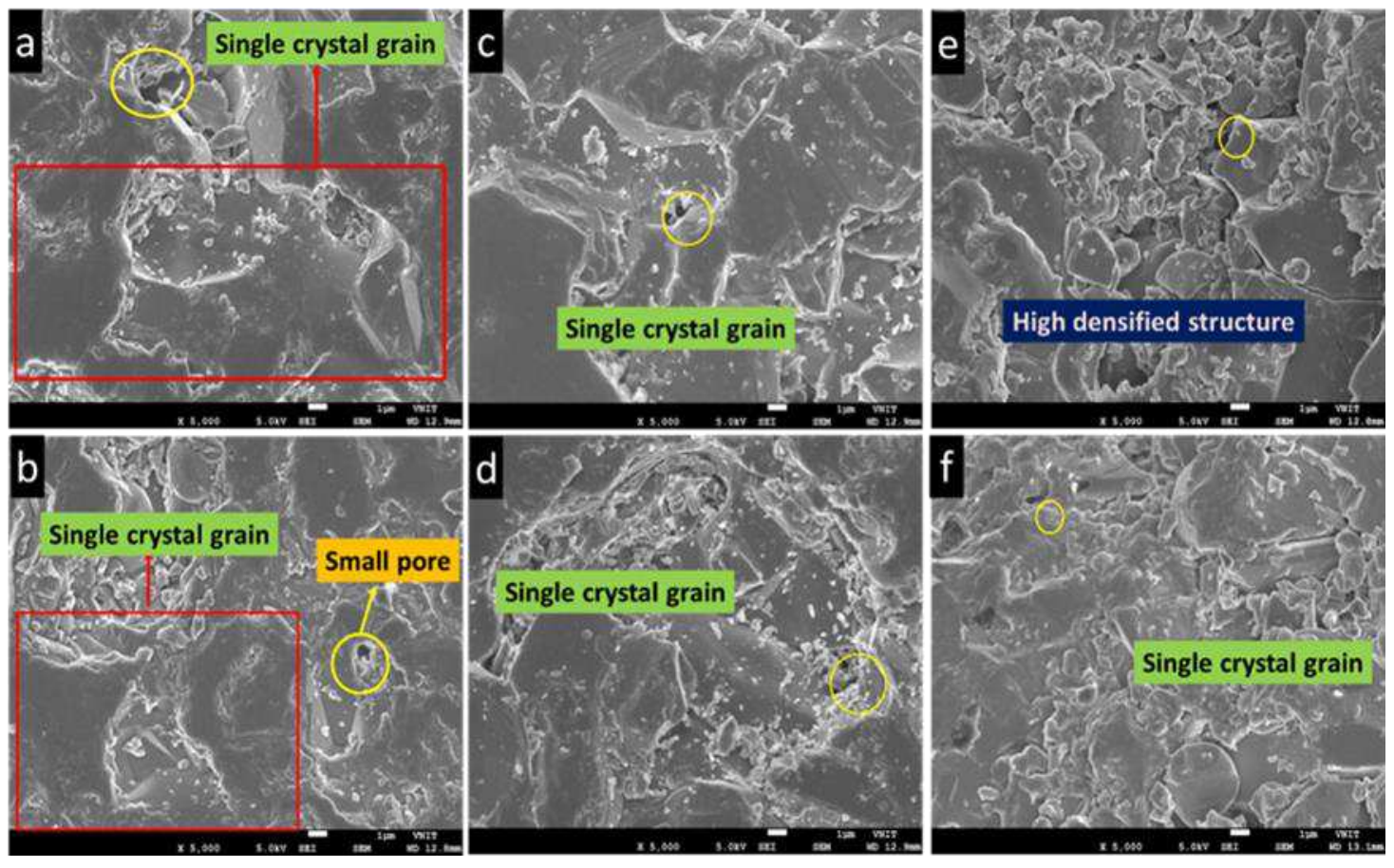

Fig. 9 FESEM micrographs of as-sintered AGPB-AZC specimen

The difference of microstructural evolution by grain diffusion mechanism in CS and AGPB sintering is schematically presented in Fig.10. The particles shown in red colour schematically represent a mixture of alumina, Zirconia, and CNT. The non-uniform heating of CS-AZC specimens causes temperature gradients across the surface. This gradient causes 
uneven diffusion processes like volume/lattice diffusion, surface diffusion, and grain boundary diffusion during grain growth, etc. Few regions which received enough activated heat energy ultimately formed densified structures with hexagonal corundum grains. However, most regions received insufficient heat energy leading to a scattered grain diffusion process which further causes highly porous ceramic structures. Such structures typically exhibit poor and scattered mechanical properties.

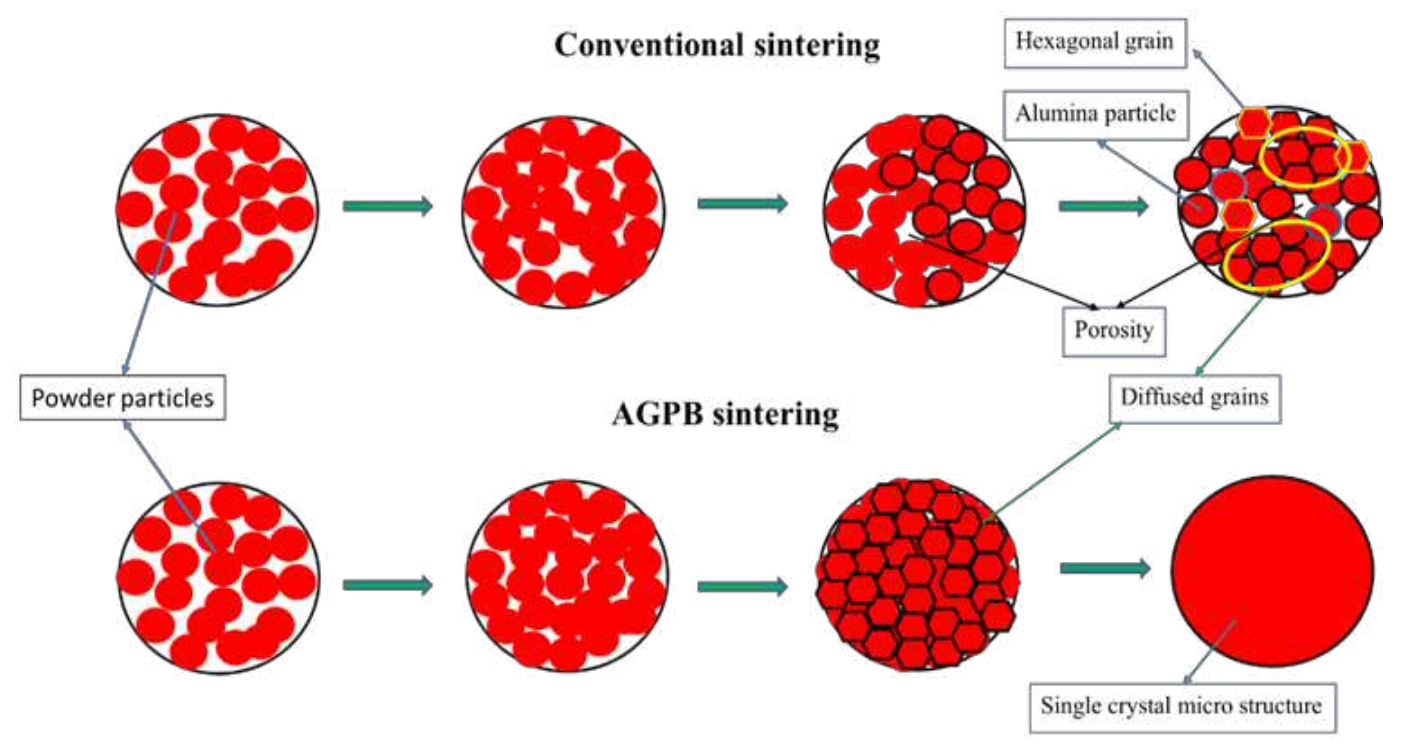

Fig. 10 Schematic of microstructural evolution in CS-AZC and AGPB-AZC sintering

However, the uniform heating in AGPB-AZC sintering allows material transport between powder particles across the open/pore spaces. Such material transport results in surface smoothing driven by specific mechanisms like evaporation and condensation, diffusion by vacancy, and viscous flow [13]. The uniform conduction heating mode throughout the sintering cycle helped in vanishing the pores across the surface. This phenomenon leads to the fabrication of a highly densified single-crystal structure in AGPB-AZC composite, as represented in Fig. 10.

The microstructural difference in CS-AZC and AGPB-AZC is shown in Fig. 11(a-b). The CS-AZC exhibited a highly porous structure as shown in Fig. 11 (a). A single crystal structure formation was confirmed in FESEM analysis of AGPB-AZC composite, as shown in Fig. 11(b). A similar single-crystal alumina structure was observed in studies by Scott et. al.[14]. They proposed a low-cost solid-state crystal conversion process where complex polycrystalline ceramics were converted to single crystals driven by an abnormal grain growth mechanism. They also reported a considerable reduction in residual porosity, which further increased relative density due to single-crystal microstructures. 

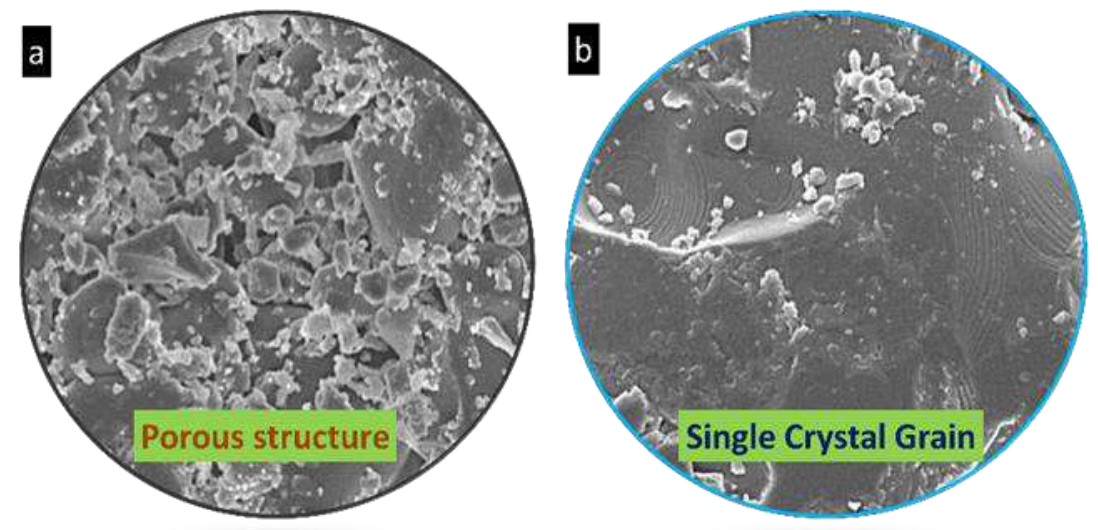

Fig. 11 FESEM micrographs of (a) CS-AZC with porous structure and (b) AGPB-AZC with a single-crystal structure

\subsection{Porosity}

The porosity difference between CS-AZC and AGPB-AZC was quantified using ImageJ software which provides a precise digital porosity measurement through porosity threshold image (this separates the porosity voids from the rest of the objects) [15]. The dark regions representing open space/pore holes were more in CS-AZC than AGPB-AZC composites, as shown in Fig. 12 (a-b).
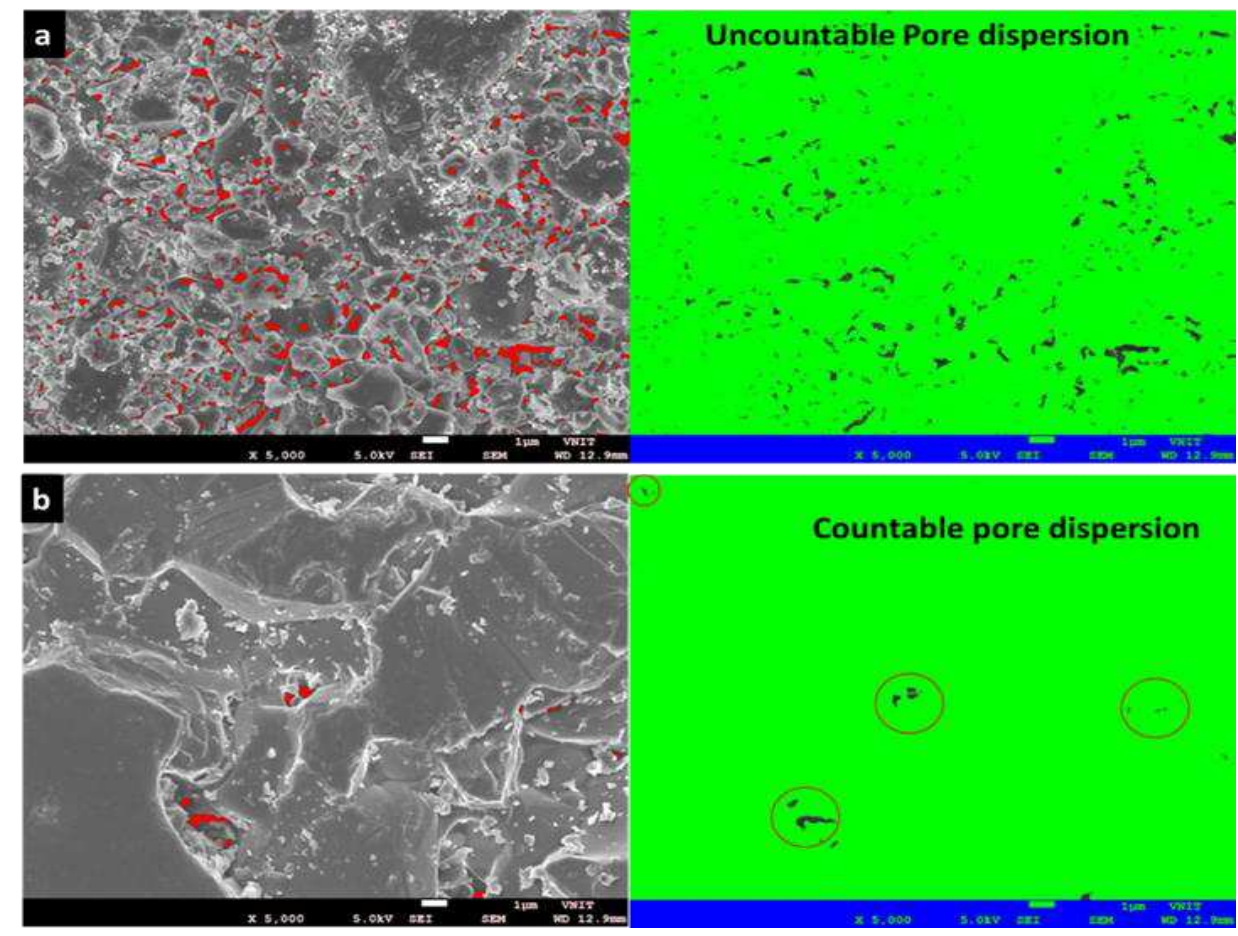

Fig. 12 Quantification of porosity in (a) CS-AZC and (b) AGPB-AZC composites

The CS-AZC specimen showed scattered pores across the surface. Small micropores were dispersed due to insufficient and non-uniform heating, as shown in Fig. 12 (a). These pores 
were further quantified in terms of pore count, average pore size, $\%$ area. The results presented further in Table 1 are the average of a minimum of ten similar micrographs.

Table 1 Porosity difference in CS-AZC and AGPB-AZC composites

\begin{tabular}{|c|c|c|c|}
\hline Sintering Technique & Average Pore Count & Average Size & Average \% Area \\
\hline CS - AZC & 110 & 0.035 & 0.8236 \\
\hline AGPB - AZC & 12 & 0.053 & 0.145 \\
\hline
\end{tabular}

A massive $89 \%$ difference in average pore count between CS-AZC and AGPB-AZC composites reflects better sinterability of the AGPB technique. Similarly, the average size and area of the pores were more in the CS-AZC composite.

\subsection{Hardness}

Across surface hardness variation in CS-AZC and AGPB-AZC was measured at twenty-four different locations, as shown in Fig 13 and 14, respectively. The locations were chosen with a $1 \mathrm{~mm}$ increment across $\mathrm{X}$ and $\mathrm{Y}$ - direction, and the detailed hardness mapping across the surface was performed. The results presented are an average of a minimum of five specimens across mutually perpendicular directions. The CS-AZC specimens showed significant hardness variation across the surface, as illustrated in Fig. 13. The hardness range varied between 1098$1386 \mathrm{HV}$ with a deviation and mean of 288 and $1273.5 \mathrm{HV}$, respectively. At the same time, a significant hardness difference was observed across $\mathrm{X}$ and $\mathrm{Y}$ directions. Such uneven hardness is associated with scattered pores due to the non-uniform heat transfer in CS-AZC. Harada et al. [16] and Abbas et al. [17] also observed Similar hardness variation. Nevarez-Rascon et al. [18] reported a similar hardness difference associated with the residual porosity level in their dental ceramic composites. They noted that the higher porosity resulted in a lower grain boundary concentration due to the formation of fewer grains than dense structures. The grain boundary formation reduces due to lower dislocation movement frequency. Consequently, the dislocation accumulates at grain boundary regions and very less energy is required for further deformation. With higher porosity in CS-AZC, the indentation energy during hardness measurement is consumed by material flow and displacement, which diminishes the apparent hardness. 


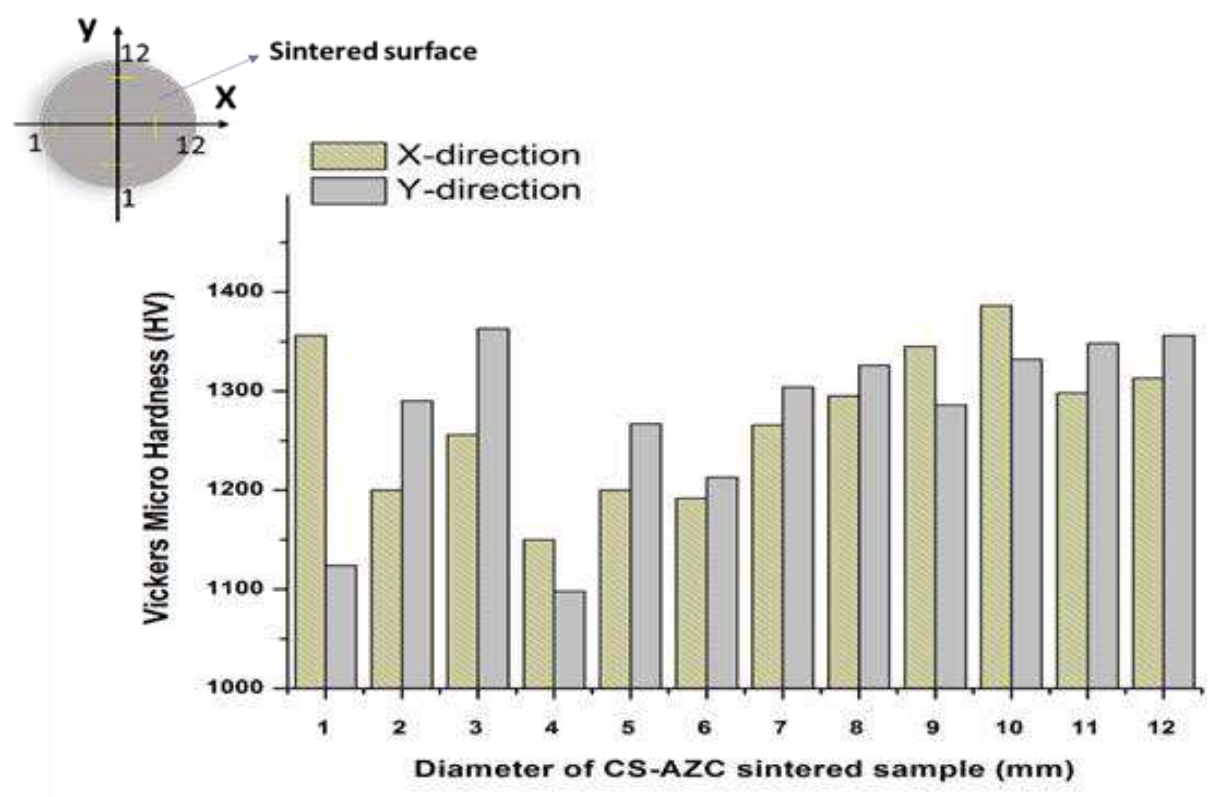

Fig. 13 Hardness of CS-AZC sintered composites

Comparatively, the AGPB-AZC specimen showed minor hardness gradients and even identical hardness across $\mathrm{X}$ and $\mathrm{Y}$ directions, as represented in Fig. 14. The maximum and minimum measured hardness was $1850 \mathrm{HV}$ and $1806 \mathrm{HV}$, respectively. The deviation and mean hardness were $44 \mathrm{HV}$ and $1829.95 \mathrm{HV}$, respectively, as shown in Table 2.

Table 2 Density and Hardness variation between CS-AZC and AGPB-AZC

\begin{tabular}{|c|c|c|c|c|c|}
\hline \multirow{2}{*}{$\begin{array}{c}\text { Sintering } \\
\text { Technique }\end{array}$} & \multicolumn{4}{|c|}{ Hardness (HV) } & \multirow{2}{*}{$\begin{array}{c}\text { Density } \\
\left(\mathrm{g} / \mathrm{cm}^{3}\right)\end{array}$} \\
\cline { 2 - 5 } & Maximum & Minimum & Deviation & Mean & 3.55 \\
\hline CS-AZC & 1386 & 1098 & 288 & 1273.5 & 3.91 \\
\hline AGPB-AZC & 1850 & 1806 & 44 & 1829.95 & \\
\hline
\end{tabular}

The uniform heating and lower porosity rate ensured consistent hardness and comparatively dense structure across surfaces. The AGPB-AZC formed dense structure was confirmed with a $10 \%$ increase in density from $3.55 \mathrm{~g} / \mathrm{cm}^{3}$ to $3.91 \mathrm{~g} / \mathrm{cm}^{3}$. 


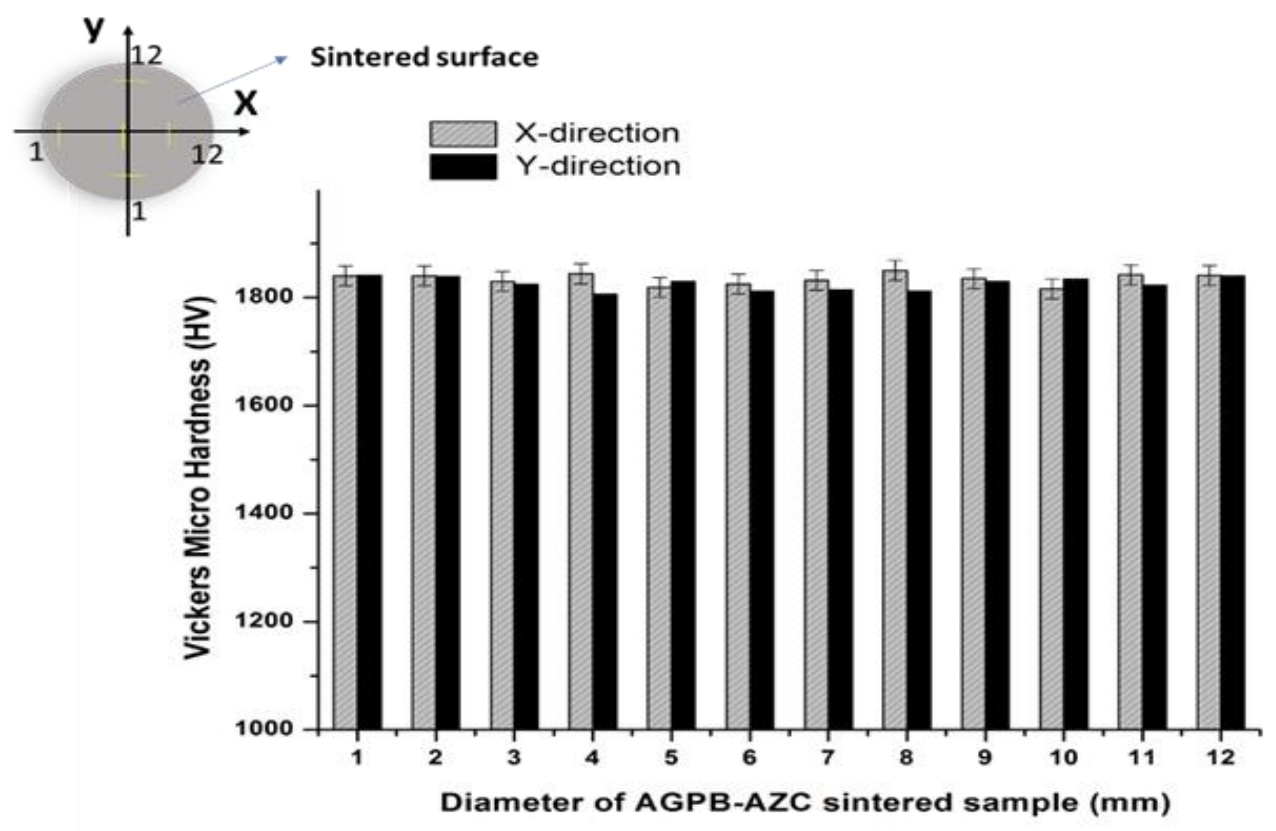

Fig.14 Hardness of AGPB-AZC sintered composites

Historically, the formation of a single-crystal structure involves a high-cost manufacturing process. Single crystals cannot be grown with optimized concentrations and uniform distribution in many unique applications like X-ray scintillators or optical lasers [16]. Hence, the current AGPB-AZC process can be advantageous and even revolutionary if the formation of a single-crystal structure can be optimized. The AGPB-AZC technique involves several critical aspects: optimizing the powder bath preparation, achieving an actual high temperature, holding the specimens at a high temperature, controlling the heat loss, maintaining a constant heat supply rate, etc. Further research on these aspects and appropriate optimization, AGPB can be a comparatively inexpensive sintering process that provides high-strength ceramic with dense single-crystal structures.

\section{Conclusions}

In this work, we proposed the usage of novel Alumina-Graphite Powder Bath (AGPB) sintering technique over the conventional sintering (CS) for fabricating Zirconia $\left(\mathrm{ZrO}_{2}\right)$ and Carbon Nano Tubes (CNTs) embedded Alumina $\left(\mathrm{Al}_{2} \mathrm{O}_{3}\right)$ composite. The fabricated AGPB-AZC composite exhibited superior mechanical and structural properties due to the addition of Zirconia and CNT compared to CS-AZC. The advantages of heat transfer mode conversion (convection-radiation to conduction) and unform heating were evidently observed through uniform hardness across the surface, minor porosity, and formation of dense single-crystal structures. The mean hardness of the AGPB-AZC (1829.95 HV) composite was enhanced by 
$43 \%$ compared to CS-AZC (1273.5 HV). The formation of single-crystal structures usually involves costlier and high-end fabrication techniques. Further research on the current practice and optimization of involved input parameters can lead to a cost-effective sintering technique capable of producing highly desirable single-crystal structured ceramics.

\section{Acknowledgment}

The authors gratefully acknowledge Director, VNIT, Nagpur and Castwel industries, MIDC, Nagpur, Maharashtra, for proving the necessary experimental setup.

\section{References}

[1] Kristen H. Brosnan, Messingand. Gary L., K. AD. Microwave Sintering of Alumina at 2.45 GHz. J $\quad$ Am Ceram Soc 2003;86:1307-12. https://doi.org/10.1016/j.jmatprotec.2007.10.057.

[2] Wang J, Raj R. Activation Energy for the Sintering of Two-Phase Alumina/Zirconia Ceramics. J Am Ceram Soc 1991;74:1959-63. https://doi.org/10.1111/j.11512916.1991.tb07815.x.

[3] Sutharsini U, Thanihaichelvan M, Singh R. Two-Step Sintering of Ceramics. Sinter. Funct. Mater., 2018, p. 3-22. https://doi.org/10.5772/68083.

[4] Gremillard L, Biotteau-Deheuvels K, Clement P, Perron M, Duvauchelle P, Meille S, et al. Modeling and in-situ evaluation of thermal gradients during sintering of large ceramic balls. Ceram Int 2017;43:7338-45. https://doi.org/10.1016/j.ceramint.2017.03.037.

[5] Green DJ, Guillon O, Rödel J. Constrained sintering: A delicate balance of scales. J Eur Ceram Soc 2008;28:1451-66. https://doi.org/10.1016/j.jeurceramsoc.2007.12.012.

[6] Fellah M, Aissani L, Abdul Samad M, Prakasam M, Purnama A, Montagne A, et al. Effect of calcination temperature on friction and wear behavior of $\alpha$-alumina $(\alpha-\mathrm{Al} 2 \mathrm{O} 3)$ for biomedical applications. Int J Appl Ceram Technol 2019;16:462-70. https://doi.org/10.1111/ijac.13116.

[7] Makireddi D, Ghuge VD, Behera A. Use of powder bath sintering for the fabrication of hard ceramic tools. Mater Manuf Process 2021. https://doi.org/10.1080/10426914.2021.1948053.

[8] Leib EW, Vainio U, Pasquarelli RM, Kus J, Czaschke C, Walter N, et al. Synthesis and thermal stability of zirconia and yttria-stabilized zirconia microspheres. J Colloid 
Interface Sci 2015;448:582-92. https://doi.org/10.1016/j.jcis.2015.02.049.

[9] Benzaid R, Chevalier J, Saâdaoui M, Fantozzi G, Nawa M, Diaz LA, et al. Fracture toughness, strength and slow crack growth in a ceria stabilized zirconia-alumina nanocomposite for medical applications. Biomaterials 2008;29:3636-41. https://doi.org/10.1016/j.biomaterials.2008.05.021.

[10] Erik T. Thostensona, Zhifeng Renb T-WC, ADepartment. Advances in the science and technology ofcarbon nanotubes and their composites: a review Erik. Compos Sci Technol 2001.

[11] Taktak R, Baklouti S, Bouaziz J. Effect of binders on microstructural and mechanical properties of sintered alumina. Mater Charact 2011;62:912-6. https://doi.org/10.1016/j.matchar.2011.06.011.

[12] Krell A. Vickers hardness and microfracture of single and polycrystalline A12O3. Krist Und Tech 1980;15:1467-74. https://doi.org/10.1002/crat.19800151229.

[13] Kang SJL, Park JH, Ko SY, Lee HY. Solid-state conversion of single crystals: The principle and the state-of-the-art. J Am Ceram Soc 2015;98:347-60. https://doi.org/10.1111/jace.13420.

[14] Scott C, Kaliszewski M, Greskovich C, Levinson L. Conversion of polycrystalline Al2O3 into single-crystal sapphire by abnormal grain growth. J. Am. Ceram. Soc., vol. 85, 2002, p. 1275-80. https://doi.org/10.1111/j.1151-2916.2002.tb00257.x.

[15] Grove C, Jerram DA. Computers \& Geosciences jPOR : An ImageJ macro to quantify total optical porosity from blue-stained thin sections. Comput Geosci 2011;37:1850-9. https://doi.org/10.1016/j.cageo.2011.03.002.

[16] Harada K, Shinya A, Yokoyama D, Shinya A. Effect of loading conditions on the fracture toughness of zirconia. J Prosthodont Res 2013;57:82-7. https://doi.org/10.1016/j.jpor.2013.01.005.

[17] Abbas MKG, Ramesh S, Lee KYS, Wong YH, Ganesan P, Alengaram UJ, et al. Effects of sintering additives on the densification and properties of alumina-toughened zirconia ceramic composites. Ceram Int 2020;46:27539-49. https://doi.org/10.1016/j.ceramint.2020.07.246.

[18] Nevarez-rascon A, Aguilar-elguezabal A, Orrantia E, Bocanegra-bernal MH. Int . Journal of Refractory Metals \& Hard Materials On the wide range of mechanical 
properties of ZTA and ATZ based dental ceramic composites by varying the Al 2 O 3 and $\mathrm{ZrO} 2$ content. Int J Refract Met Hard Mater 2009;27:962-70. https://doi.org/10.1016/j.ijrmhm.2009.06.001. 